Opt i cal emi ssi on from a hi gh- ref r act i ve- i ndex wavegui de excited by a travel ing el ectron beam

\begin{tabular}{|l|l|}
\hline 著者 & $\begin{array}{l}\text { Kuwamur a Yuj i , Yamada Mnor u, Okanto o Ryui chi , } \\
\text { Kanai Takeshi , Far es Hesham }\end{array}$ \\
\hline $\begin{array}{l}\text { j our nal or } \\
\text { publ i cat i on ti tl e }\end{array}$ & Journal of Appl i ed Physi cs \\
\hline vol une & 104 \\
\hline nunber & 10 \\
\hline page range & 103105 \\
\hline year & 2008 01- 01 \\
\hline URL & ht t p: //hdl . handl e. net /2297/12581 \\
\hline
\end{tabular}




\title{
Optical emission from a high-refractive-index waveguide excited by a traveling electron beam
}

\author{
Yuji Kuwamura, ${ }^{\text {a) }}$ Minoru Yamada, Ryuichi Okamoto, Takeshi Kanai, and Hesham Fares \\ Division of Electrical Engineering and Computer Science, Graduate School of Natural Science and \\ Technology, Kanazawa University, Kakuma-machi, Kanazawa 920-1192, Japan
}

(Received 17 January 2008; accepted 4 October 2008; published online 18 November 2008)

\begin{abstract}
An optical emission scheme was demonstrated, in which a high-refractive-index waveguide is excited by a traveling electron beam in a vacuum environment. The waveguide was made of $\mathrm{Si}-\mathrm{SiO}_{2}$ layers. The velocity of light propagating in the waveguide was slowed down to $1 / 3$ of that in free space due to the high refractive index of Si. The light penetrated partly into the vacuum in the form of a surface wave. The electron beam was emitted from an electron gun and propagated along the surface of the waveguide. When the velocity of the electron coincided with that of the light, optical emission was observed. This emission is a type of Cherenkov radiation and is not conventional cathode luminescence from the waveguide materials because $\mathrm{Si}$ and $\mathrm{SiO}_{2}$ are transparent to light at the emitted wavelength. This type of emission was observed in an optical wavelength range from 1.2 to $1.6 \mu \mathrm{m}$ with an electron acceleration voltage of $32-42 \mathrm{kV}$. The characteristics of the emitted light, such as the polarization direction and the relation between the acceleration voltage of the electron beam and the optical wavelength, coincided well with the theoretical results. The coherent length of an electron wave in the vacuum was confirmed to be equal to the electron spacing, as found by measuring the spectral profile of the emitted light.

(c) 2008 American Institute of Physics. [DOI: 10.1063/1.3021150]
\end{abstract}

\section{INTRODUCTION}

Lasers are the principal devices used in optoelectronics and have contributed to the development of optical communication systems and optical storage technology. Conventional lasers utilize electronic transitions between the energy levels inherent in specific gases, solids, semiconductors, or organic molecules. This means that the operating wavelength of the laser is restricted by the energy levels in each material.

Moreover, the optical amplification in a conventional laser is symmetric for forward and backward propagations of the optical light. Once the light emitted from a laser is reflected by the surface of a connected optical device and reinjected into the laser, the reinjected light is amplified, resulting in the unstable operation of the laser. Successful developments in electronics such as the achievement of information processing and the construction of complicated circuits rely on the unidirectional property of electron tubes and transistors. This property provides suitable unidirectional amplification from the input port to the output port but prevents the propagation of the signal in the opposite direction.

There are several devices that enable the unidirectional amplification of electromagnetic waves, such as the traveling-wave tube, ${ }^{1,2}$ the free electron laser, ${ }^{3,4}$ and the Cherenkov laser. ${ }^{5-20}$ These devices utilize the kinetic energy of an electron beam in a vacuum, and the condition required to obtain the radiation and to amplify the electromagnetic wave is that the velocities of the electron beam and the electromagnetic wave coincide. Therefore, these devices can op-

\footnotetext{
${ }^{a}$ Author to whom correspondence should be addressed. Tel.: 81-76-2344867. FAX: 81-76-234-4870. Electronic mail: kuwamura@t.kanazawau.ac.jp.
}

erate, in principle, over a very wide frequency range extending from the microwave to the $\mathrm{x}$-ray regions of the electromagnetic spectrum.

The traveling-wave tube operates at a lower voltage of several kilovolts, but its operation at wavelengths shorter than the submillimeter region is difficult because of limitations in the precision of the fabrication technology used to form the helix coil. ${ }^{1,2}$ The free electron laser can operate in a wide frequency range from the microwave to the $\mathrm{x}$-ray regions, but it requires a very high acceleration voltage exceeding 10-1000 MV and a large experimental setup.,4

The Cherenkov laser has the possibility of operation at a relatively low voltage with a compact size. ${ }^{12-21}$ Radiation of an electromagnetic wave at approximately $100 \mathrm{GHz}$ from a Cherenkov laser with an electron acceleration voltage of $35-75 \mathrm{kV}$ has been reported. ${ }^{19}$

However, radiation in the optical frequency range obtained from a Cherenkov laser has not yet been observed. We had theoretically proposed a scheme for optical amplification using a traveling electron beam and a high-refractive-index waveguide in a vacuum. ${ }^{22,23}$ Our proposed scheme involves a type of Cherenkov laser, but our proposal was analyzed on the basis of a quantum mechanical treatment which is not the same as many theories already established on the Cherenkov laser. An important parameter in the quantum mechanical treatment is the spreading width or the coherent length of a single electron. This parameter has not been introduced in the classical theory because the electron is assumed to be a point particle.

We also experimentally observed the optical amplification for an incident light injected through an optical fiber from the outside of a vacuum chamber. ${ }^{24}$ However, we could 


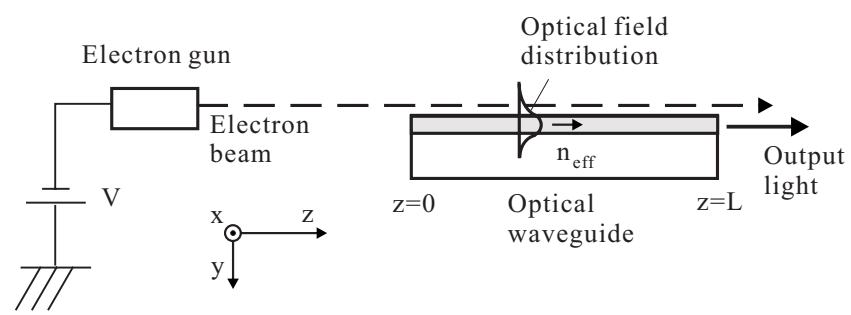

FIG. 1. Configuration of an optical emitter consisting of a high-refractiveindex optical waveguide and an electron gun. The optical light propagates in the waveguide with decreasing velocity and penetrates partly into the vacuum region.

not obtain detailed information on the amplification such as the coherent length of the electron wave because the experiment was adversely affected by the accumulation of contaminants in the vacuum chamber because we attached the input optical fiber with an adhesive material.

In this study, we demonstrate the observation of optical emission from a high-refractive-index optical waveguide excited by a traveling electron beam without any input light. This experiment is more stable than our previous one, ${ }^{24}$ and we were able to estimate the coherent length of an electron wave in a vacuum by comparing the theoretical results with the experimental data.

In Sec. II, the mechanisms of optical emission and amplification are briefly reviewed. In Sec. III, a theoretical model based on a quantum mechanical treatment is given to predict the optical emission. In Sec. IV, the experimental setup and the observed results are discussed. In Sec. V, we compare the experimental data with the theoretical results. In Sec. VI, our theoretical results are compared with the classical treatment. Similarities between the classical and quantum mechanical treatments are discussed. The conclusions of this paper are given in Sec. VII.

\section{CONFIGURATION OF DEVICE AND MECHANISM OF OPTICAL EMISSION}

The proposed configuration for the optical emission scheme is illustrated in Fig. 1, where an optical waveguide with a high refractive index and an electron gun are installed in a vacuum chamber. An electron beam is emitted from the electron gun and is adjusted to propagate along the surface of the optical waveguide in the $z$ direction. The optical field propagates through the waveguide in the $z$ direction with decreasing velocity due to the high refractive index of the waveguide material, and penetrates partly into the vacuum region in the form of a surface wave or an evanescent wave. An optical field is generated and amplified by the electron beam when the velocity $v_{e}$ of the electron becomes slightly faster than the phase velocity $v_{\text {opt }}$ of the guided optical light.

The optical emission is explained in a quantum mechanical manner in terms of electron transitions using an energy $E$ versus momentum $p$ diagram as shown in Fig. 2. We suppose that the energy, momentum, and wave number of an electron at level $b$ emitted by the electron gun are $E_{b}, p_{b}$, and $k_{b}$, respectively. This electron transits to level $a$ by interaction with an optical field, with an angular frequency of $\omega$ and a propagation constant of $\beta$ in the $z$ direction.

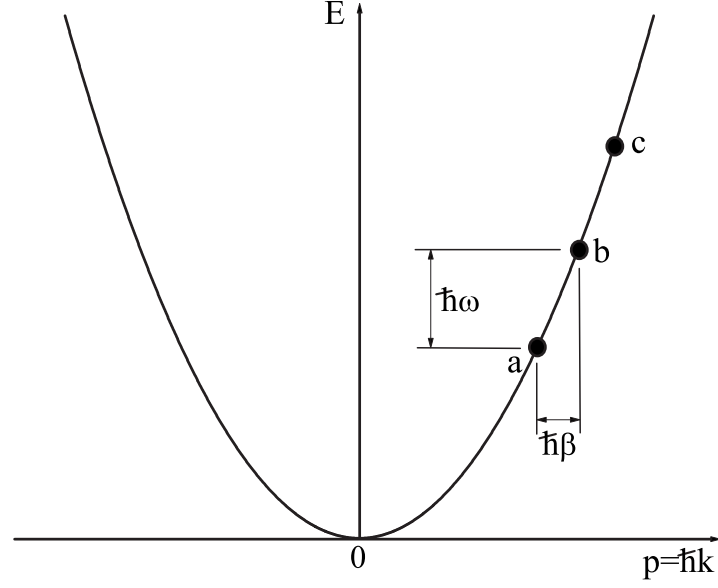

FIG. 2. Dispersion relation between the energy $E$ and the momentum $p$ of an electron. The electron emitted by the electron gun is at level $b$ and transits by optical emission to level $a$. Both energy and momentum conservation rules must be obeyed.

Here, the energy and momentum conservation rules should be obeyed, ${ }^{22,23,25}$

$$
\begin{aligned}
& E_{b}-E_{a}=\hbar \omega, \\
& p_{b}-p_{a}=\hbar\left(k_{b}-k_{a}\right)=\hbar \beta .
\end{aligned}
$$

The velocity $v_{e}$ of the electron wave is defined as the partial derivative of the energy $E$ with respect to the momentum $p=\hbar k$. Since the energy difference between levels $b$ and $a$ is sufficiently small compared with the corresponding energy $E_{b}$ or $E_{a}$, we obtain

$$
v_{e}=\frac{\partial E}{\partial p}=\frac{E_{b}-E_{a}}{\hbar\left(k_{b}-k_{a}\right)}=\frac{\omega}{\beta} .
$$

Here $\omega / \beta=v_{\mathrm{opt}}$ is the phase velocity of the optical field. Clearly, Eq. (3) gives the condition

$$
v_{e}=v_{\mathrm{opt}} \text {, }
$$

which is also known as the condition for obtaining Cherenkov radiation. ${ }^{25}$

Conventional Cherenkov lasers utilize a cylindrical dielectric waveguide, whose refractive index is approximately 1.2 , to decrease the velocity $v_{\text {opt }}$ of the electromagnetic wave to that of the electron beam, $v_{e} \cdot{ }^{5-10}$ However, in our scheme, a slab waveguide made of a semiconductor crystal with a high refractive index, such as 3.5 , is used to more effectively reduce $v_{\text {opt }}$.

Another condition required for the electron transition is that the optical light must have an electric-field component in the propagation direction of the electron beam. In the case of the slab waveguide shown in Fig. 1, transverse magnetic (TM) modes can be generated and amplified because the TM modes have a field component $E_{z}$ in addition to components $H_{x}$ and $E_{y}$. Transverse electric (TE) modes should be neither generated nor amplified because the TE modes consist of the components $E_{x}, H_{y}$, and $H_{z}$.

An electron at level $b$ can also transit to a higher level $c$ while satisfying both the energy and momentum conservation rules, resulting in optical absorption. In this case, the 
optical gain for the light amplification is given as the difference between the electron transition probability from level $b$ to level $a$ and that from level $b$ to level $c$. The optical gain becomes positive when the velocity of the electron, $v_{e}$, is slightly faster than the phase velocity of the optical field, $v_{\text {opt }}$, as will be shown in Sec. III.

The probability of an electron transition from an upper level to a lower level is proportional to $S+1$, while that from a lower level to an upper level is proportional to $S$, where $S$ is the photon number. Then, an electron can transit to a lower level even when $S=0$, which is called spontaneous emission. Since the spontaneous emission is generated by the existence of a so-called zero-point field, whose quantum number is $1 / 2$, it has an identical field distribution to the measurable optical field. Hence, the spontaneous emission in our proposed device should be limited to a specific TM mode that satisfies the relation $v_{e}=v_{\mathrm{opt}}$. Therefore, we call this emission "guided spontaneous emission" to distinguish it from the conventional spontaneous emission, which is radiated in all directions in space and in all polarization directions.

\section{THEORETICAL APPROACH}

\section{A. Variation in the optical field}

We start this analysis by following a semiclassical treatment, where the optical field is represented in a classical manner but the dynamics of the electron is described in terms of quantum mechanics. After that, we introduce the spontaneous emission by comparing the classical optical field with the quantum mechanical treatment of the optical field.

The effects of the traveling electron are introduced in the classical field equation with current densities of $\boldsymbol{J}_{\mathrm{gsp}}$ for the guided spontaneous emission and $\boldsymbol{J}_{\mathrm{st}}$ for the stimulated emission. These current densities are quantum mechanical operators. The variations in a guided optical field $\boldsymbol{E}$ are given by the classical wave equation

$$
\nabla^{2} \boldsymbol{E}-\mu_{0} \sigma \frac{\partial \boldsymbol{E}}{\partial t}-\mu_{0} \varepsilon(x, y) \frac{\partial^{2} \boldsymbol{E}}{\partial t^{2}}=\mu_{0} \frac{\partial\left(\boldsymbol{J}_{\mathrm{gsp}}+\boldsymbol{J}_{\mathrm{st}}\right)}{\partial t},
$$

where $\varepsilon(x, y)$ is a dielectric constant including structural information of the waveguide as a function of $x$ and $y$, and $\sigma$ is the conductivity representing the loss mechanism in the waveguide such as free electron absorption. We suppose here that the optical field propagates in the $+z$ direction in the form of

$$
\boldsymbol{E}=F(z) \boldsymbol{T}(x, y) e^{j(\omega t-\beta z)}+\text { c.c. },
$$

where $F(z)$ is the field amplitude and $\boldsymbol{T}(x, y)$ is the transverse-field distribution function characterized by

$$
\left[\nabla^{2}+\mu_{0} \varepsilon(x, y) \omega^{2}\right] \boldsymbol{T}(x, y) e^{j \beta z}=0,
$$

with a normalization in the form of

$$
\begin{aligned}
\int_{-\infty}^{\infty} \int_{-\infty}^{\infty}|\boldsymbol{T}(x, y)|^{2} d x d y= & \int_{-\infty}^{\infty} \int_{-\infty}^{\infty}\left[\left|T_{x}(x, y)\right|^{2}+\left|T_{y}(x, y)\right|^{2}\right. \\
& \left.+\left|T_{z}(x, y)\right|^{2}\right] d x d y=1
\end{aligned}
$$

The propagation constant $\beta$ and the field distribution function are analyzed as an eigenvalue and an eigenfunction, respectively, for a given structure of the waveguide via Eq. (7). The propagation constant $\beta$ corresponds to the phase velocity of the optical light. We define the effective refractive index $n_{\text {eff }}$ using the relation

$$
\beta=\sqrt{\mu_{0} \varepsilon_{0}} \omega n_{\mathrm{eff}}=\omega \frac{n_{\mathrm{eff}}}{c}=\frac{2 \pi n_{\mathrm{eff}}}{\lambda},
$$

where $\lambda$ is the wavelength in the free space. Namely, the phase velocity $v_{\text {opt }}$ of the guided mode is related to $n_{\text {eff }}$ as

$$
v_{\mathrm{opt}}=\frac{\omega}{\beta}=\frac{c}{n_{\mathrm{eff}}} .
$$

Here, we write the expectation value of a quantum mechanical operator or a spatial function $A$ as $\langle A\rangle$. By multiplying both sides of Eq. (5) by $\boldsymbol{T}^{*}(x, y) \exp j(\beta z-\omega t)$ and by taking the expectation value for the spatial and time averages, the variation in the field amplitude along the $z$ direction is given as $^{22,23}$

$$
\begin{aligned}
\frac{d F(z)}{d z}= & -\frac{\mu_{0} \omega}{2 \beta} \int_{B}\left\langle\left(\boldsymbol{J}_{\mathrm{gsp}}+\boldsymbol{J}_{\mathrm{st}}\right) \cdot \boldsymbol{T}^{*}(x, y) e^{j \beta z}\right\rangle d^{2} \boldsymbol{r} e^{-j \omega t} \\
& -\frac{\alpha_{\text {loss }}}{2} F(z),
\end{aligned}
$$

where $\int_{B} d^{2} \boldsymbol{r}$ indicates two-dimensional integration over a cross-sectional area $B$ of the electron beam.

The current density $\boldsymbol{J}_{\text {st }}$ has a component that vibrates with the existing optical field, while the other current density $J_{\text {gsp }}$ is not directly affected by the optical field. Then Eq. (11) is rewritten as

$$
\frac{d|F(z)|^{2}}{d z}=F^{*} \frac{d F}{d z}+F \frac{d F^{*}}{d z}=\left(g-\alpha_{\mathrm{loss}}\right)|F(z)|^{2}+C_{\mathrm{gsp}},
$$

where $g$ is the gain coefficient,

$$
g=-\frac{\mu_{0} \omega}{\beta} \operatorname{Re}\left\{\frac{1}{F} \int_{B}\left\langle\boldsymbol{J}_{\mathrm{st}} \cdot \boldsymbol{T}^{*}(x, y) e^{j \beta z}\right\rangle d^{2} \boldsymbol{r} e^{-j \omega t}\right\},
$$

$\alpha_{\text {loss }}$ is the guiding loss coefficient given by

$$
\alpha_{\mathrm{loss}}=\sqrt{\frac{\mu_{0}}{\varepsilon_{0}}} \frac{1}{n_{\mathrm{eff}}} \int_{-\infty}^{\infty} \sigma|\boldsymbol{T}(x, y)|^{2} d^{2} \boldsymbol{r}
$$

and $C_{\mathrm{gsp}}$ indicates the inclusion of the guided spontaneous emission,

$$
C_{\mathrm{gsp}}=-\frac{\mu_{0} \omega}{\beta} \operatorname{Re}\left\{F^{*} \int_{B}\left\langle\boldsymbol{J}_{\mathrm{gsp}} \cdot \boldsymbol{T}^{*}(x, y) e^{j \beta z}\right\rangle d^{2} \boldsymbol{r} e^{-j \omega t}\right\} .
$$

The gain coefficient $g$ in Eq. (13) and the term $C_{\text {gsp }}$ in Eq. (15) appear to have negative values. However, they can maintain positive values as will be determined in Secs. III B and III C.

The optical output from a device with length $L$ is obtained from Eq. (12) to be 


$$
|F(L)|^{2}=\frac{C_{\mathrm{gsp}}}{g-\alpha_{\mathrm{loss}}}\left\{e^{\left(g-\alpha_{\mathrm{loss}}\right) L}-1\right\} .
$$

This equation implies that the source of optical emission is the guided spontaneous emission given by the term $C_{\mathrm{gsp}}$, which is amplified by the stimulated emission determined by the gain coefficient $g$.

\section{B. Gain coefficient}

The gain coefficient $g$ in Eq. (13) can be evaluated using the density matrix $\rho$ similarly to the analysis in Ref. 22 as follows. The current density $\boldsymbol{J}_{\text {st }}$ is given by the absolute charge $e$ and the rest mass $m_{0}$ of an electron, the electron density $N$, the momentum operator $\boldsymbol{P}$, and the vector potential $\boldsymbol{A}$ as

$$
\boldsymbol{J}_{\mathrm{st}}=-\frac{e}{m_{0}} N(\boldsymbol{P}+e \boldsymbol{A}) \approx-\frac{e N \boldsymbol{P}}{m_{0}} .
$$

The expectation value is written as

$$
\begin{aligned}
\left\langle\boldsymbol{J}_{\mathrm{st}}\right. & \left.\cdot \boldsymbol{T}^{*}(x, y) e^{j \beta z}\right\rangle \\
& =-\frac{e N_{t}}{m_{0}} \operatorname{Tr}\left\{\rho \boldsymbol{P} \cdot \boldsymbol{T}^{*}(x, y) e^{j \beta z}\right\} \\
& =-\frac{e N_{t}}{m_{0}} \sum_{n} \int_{\ell^{3}} \varphi_{n}^{*}(\boldsymbol{r}) \rho \boldsymbol{P} \cdot \boldsymbol{T}^{*}(x, y) e^{j \beta z} \boldsymbol{\varphi}_{n}(\boldsymbol{r}) d^{3} \boldsymbol{r},
\end{aligned}
$$

where $N_{t}$ is the spatially averaged electron density over the electron beam and $\operatorname{Tr}\{\}$ indicates a quantum statistical operation called "trace" as shown in the second line in Eq. (18). $\varphi_{n}(\boldsymbol{r})$ is the electron wave function at energy level $n$ given as

$$
\varphi_{n}(\boldsymbol{r})=\sqrt{\frac{1}{\ell^{3}}} e^{j k_{n} r},
$$

where $\ell$ is the spreading length of the electron wave and is regarded as the coherent length of the electron wave because the phase variation is expected to vary in the form $\boldsymbol{k}_{n} \boldsymbol{r}$ without any phase distortion in the given volume $\ell^{3}$.

Note that the length $\ell$ in this paper is defined for a single electron and is not the full length of the proposed device $L$ shown in Fig. 1. Although we did not take into account the difference between $\ell$ and $L$ in our previous papers of Refs. 22 and 23 , our model becomes more generalized by treating $\ell$ and $L$ as different lengths.

By determining both the diagonal and off-diagonal elements of the density matrix $\rho$ as in Ref. 22 , the gain coefficient is given by

$$
g=\sqrt{\frac{\mu_{0}}{\varepsilon_{0}}} \frac{e J \tau v_{e}}{n_{\mathrm{eff}} \hbar \omega} \xi D_{\mathrm{st}}=\frac{e J \tau}{\varepsilon_{0} n_{\mathrm{eff}}^{2} \hbar \omega} \xi D_{\mathrm{st}},
$$

where $J$ is the dc current density of the electron beam, $\tau$ is the relaxation time of the electron wave, and $\xi$ is the spatial coupling coefficient between the optical field $E_{z}$ and the electron beam. ${ }^{22} D_{\text {st }}$ is a dispersion function indicating the difference between the stimulated emission upon the transition from level $b$ to level $a$ and the absorption upon the transition from level $b$ to level $c$,

$$
D_{\mathrm{st}}=\operatorname{Sinc}^{2}\left[\frac{\left(k_{b}-k_{a}-\beta\right) \ell}{2}\right]-\operatorname{Sinc}^{2}\left[\frac{\left(k_{c}-k_{b}-\beta\right) \ell}{2}\right] .
$$

In Eq. (20), the relation $v_{e} \approx c / n_{\text {eff }}=1 /\left(\sqrt{\mu_{0} \varepsilon_{0}} n_{\text {eff }}\right)$ is used.

\section{Guided spontaneous emission}

The evaluation of $C_{\mathrm{gsp}}$ in Eq. (15) requires careful treatment because the spontaneous emission cannot be derived directly from the classic optical field. We introduce the spontaneous emission by adjusting the classical treatment to the result of the field quantization.

The electron transition from level $b$ to level $a$ in Fig. 2 is given as

$$
\rho_{b b}\left(1-\rho_{a a}\right)(S+1) M_{a b} \approx \rho_{b b} M_{a b}(S+1),
$$

while the electron transition from level $b$ to level $c$ is

$$
\rho_{b b}\left(1-\rho_{c c}\right) S M_{c b} \approx \rho_{b b} M_{c b} S,
$$

where $\rho_{n n}$ is a diagonal element of the density matrix $\rho$ and $M_{m n}$ is a coefficient characterizing the transition from level $n$ to level $m$. We have supposed that the numbers of electrons located at levels $a$ and $c$ are very small; that is, $\rho_{a a} \approx \rho_{c c}$ $\approx 0$.

In Eqs. (22) and (23), the terms proportional to the photon number $S$ correspond to the simulated emission and absorption, respectively. The other term independent of the photon number is the spontaneous emission. The gain coefficient $g$ in Eq. (20) satisfies the relation

$$
g \propto\left(\rho_{b b}-\rho_{a a}\right) M_{a b}-\left(\rho_{b b}-\rho_{c c}\right) M_{c b} \approx \rho_{b b}\left(M_{a b}-M_{c b}\right) .
$$

As the next step, we determine the photon number by evaluating the optical energy. The optical field is confined in the transverse $x$ and $y$ directions, but propagates along the longitudinal $z$ direction. Then, we consider a momentary region with length $L_{f}$ and evaluate the optical energy within this region. This length $L_{f}$ is much longer than the optical wavelength, but the amplitude $F(z)$ is regarded as constant within this region. The photon number $S(z)$ in this region is determined by evaluating the optical energy as

$$
\begin{aligned}
\hbar \omega S(z) & =\int_{z-L_{f} / 2}^{z+L_{f} / 2} \int_{-\infty}^{\infty} \int_{-\infty}^{\infty} \varepsilon(x, y)|\boldsymbol{E}|^{2} d x d y d z \\
& \approx 2 \varepsilon_{0} n_{\mathrm{eff}}^{2}|F(z)|^{2} L_{f} .
\end{aligned}
$$

We suppose that the field has a periodic boundary condition in the phase variation of each length $L_{f}$,

$$
\beta_{m} L_{f}=\sqrt{\mu_{0} \varepsilon_{0}} n_{\mathrm{eff}} \omega_{m} L_{f}=2 m \pi,
$$

where $m$ is an integer indicating the mode number in the longitudinal direction.

By setting

$$
\Delta \omega=\omega_{m+1}-\omega_{m},
$$

$L_{f}$ is written as 


$$
L_{f}=\frac{2 \pi}{\sqrt{\mu_{0} \varepsilon_{0}} n_{\mathrm{eff}} \Delta \omega} .
$$

Then Eq. (12) is represented as an expression containing the photon number $S(z)$ as

$$
\frac{d|F(z)|^{2}}{d z}=\left(g-\alpha_{\text {loss }}\right) \sqrt{\frac{\mu_{0}}{\varepsilon_{0}}} \frac{\hbar \omega \Delta \omega S(z)}{4 \pi n_{\mathrm{eff}}}+C_{\mathrm{gsp}} .
$$

As found from Eqs. (22)-(24), $C_{\text {gsp }}$ should have the same coefficient as that for the stimulated emission. We thus obtain

$$
C_{\mathrm{gsp}}=\sqrt{\frac{\mu_{0}}{\varepsilon_{0}}} \frac{e J \tau \Delta \omega}{4 \pi \varepsilon_{0} n_{\mathrm{eff}}^{3}} \xi D_{\mathrm{gsp}},
$$

where $D_{\mathrm{gsp}}$ is the dispersion function for the guided spontaneous emission,

$$
D_{\mathrm{gsp}}=\operatorname{Sinc}^{2}\left[\frac{\left(k_{b}-k_{a}-\beta\right) \ell}{2}\right] .
$$

The term $\Delta \omega$ in Eq. (30) is inversely proportional to the length $L_{f}$ in Eq. (28). This relation means that $C_{\mathrm{gsp}}$ indicates the inclusion of the spontaneously emitted optical energy per unit length. However, the expression containing $\Delta \omega$ is more convenient because $\Delta \omega$ can be regarded as the measured frequency width or the measured resolution in the experimental observation of the continuous optical spectrum.

\section{Modification by relativistic treatment}

The present analysis is based on a nonrelativistic quantum mechanical treatment. When the accelerated voltage $V$ is not so high, the electron wave number $k$ and the electron velocity $v_{e}$ of the accelerated electron are simply given by

$$
k=\frac{m_{0} v_{e}}{\hbar}=\frac{\sqrt{2 m_{0} e V}}{\hbar} .
$$

However, the velocity should be determined in the frame of the relativistic treatment when the accelerated voltage $V$ is sufficiently high. We take into account the relativistic effect on the velocity and wave number using the relation $W^{2}$ $=\left(m_{0} c^{2}\right)^{2}+(c P)^{2}$, where $W$ is the total energy of an electron including the rest energy and $P$ is the momentum of the electron, $P=\hbar k=m_{0} v_{e} / \sqrt{1-\left(v_{e} / c\right)^{2}}$. Then, the wave numbers at levels $b, a$, and $c$ are given by

$$
k_{b}=\frac{1}{\hbar} \sqrt{2 m_{0} e V+\left(\frac{e V}{c}\right)^{2}}
$$

and

$$
k_{a / c}=\frac{1}{\hbar} \sqrt{2 m_{0}(e V \mp \hbar \omega)+\left(\frac{e V \mp \hbar \omega}{c}\right)^{2}} .
$$

The condition $v_{e}=v_{\mathrm{opt}}$ in Eq. (4) is given as

$$
v_{e}=c \sqrt{1-\frac{1}{\left(1+e V / m_{0} c^{2}\right)^{2}}}=\frac{c}{n_{\mathrm{eff}}}=v_{\mathrm{opt}} .
$$

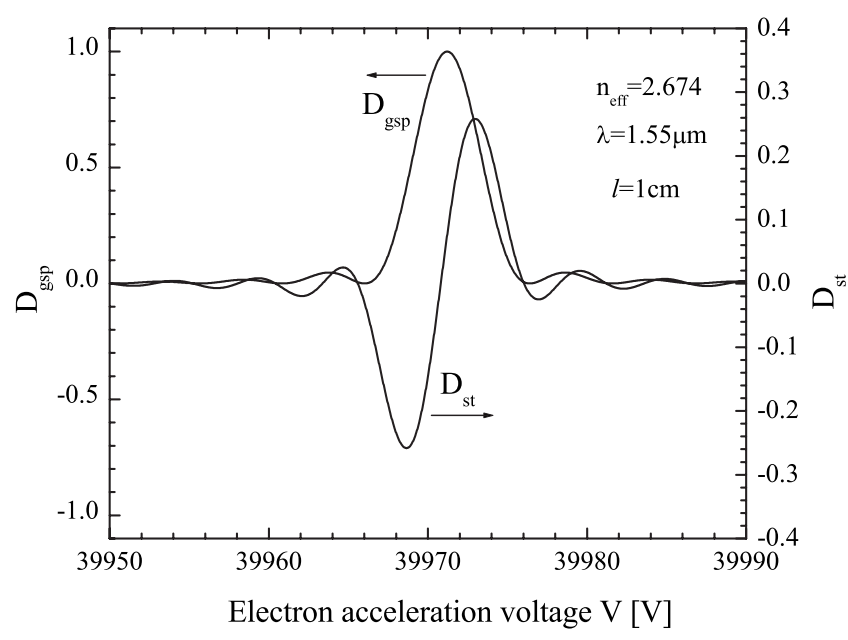

FIG. 3. Dispersion functions $D_{\mathrm{st}}$ and $D_{\mathrm{gsp}}$ for sufficiently long electron spreading length $\ell$.

\section{E. Numerical examples of the dispersion functions and emitted spectra}

Numerical examples of $D_{\mathrm{st}}$ in Eq. (21) and $D_{\mathrm{gsp}}$ in Eq. (31) are shown in Figs. 3, 4(a), and 4(b). As found from Eqs. (21) and (31), $D_{\text {st }}$ can vary in the range from -1 to 1 , and
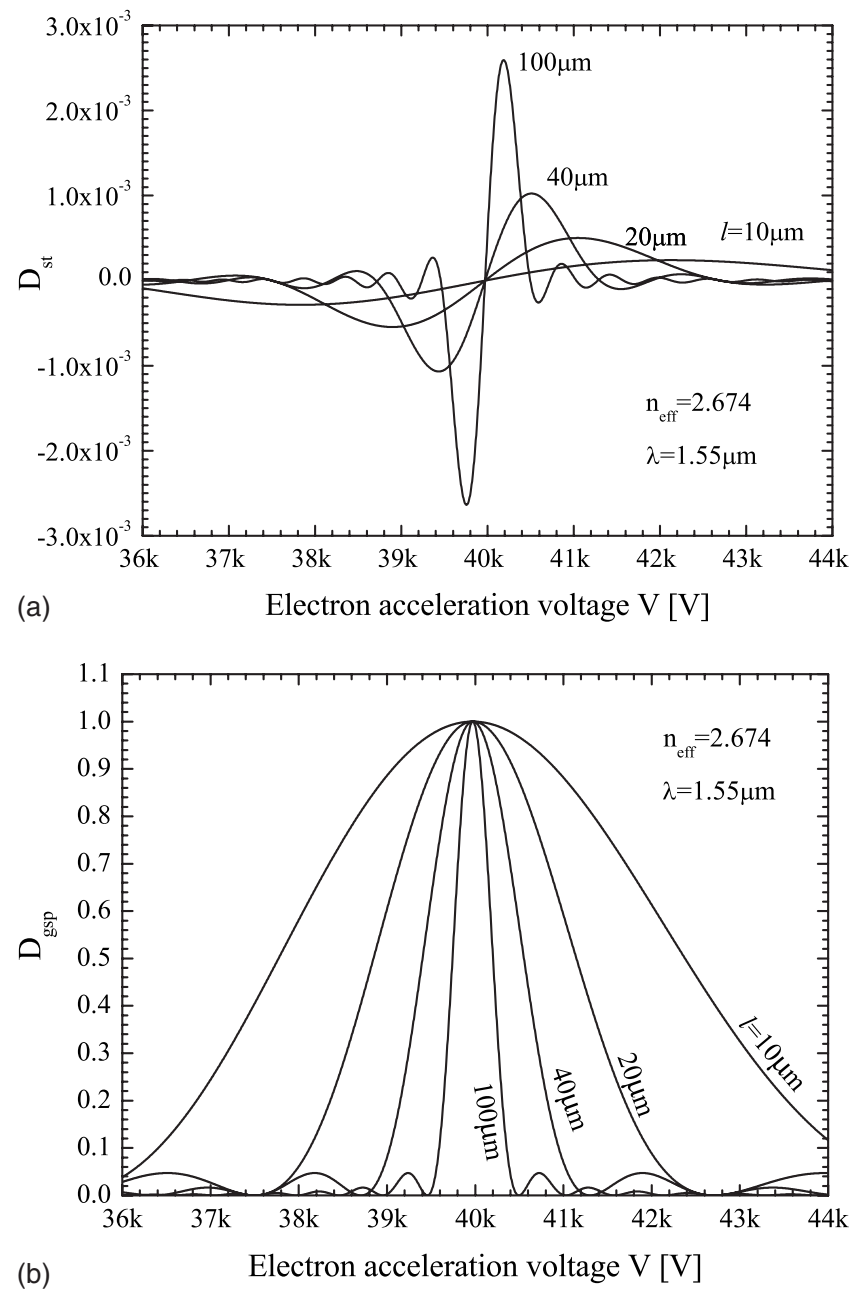

(b)

FIG. 4. Dispersion functions $D_{\mathrm{st}}$ and $D_{\mathrm{gsp}}$ for short electron spreading length $\ell$. (a) Dispersion function $D_{\mathrm{st}} . D_{\mathrm{st}}$ becomes very small when $\ell$ is small. (b) Dispersion function $D_{\mathrm{gsp}}$. 


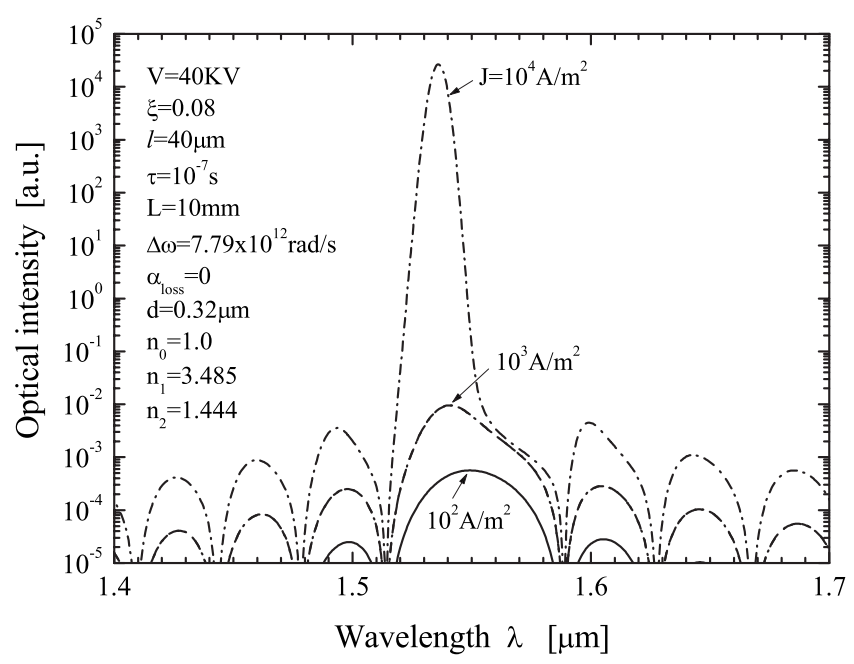

FIG. 5. Numerical examples of the optical output profile $|F(L)|^{2}$. The profile coincides with that of $D_{\text {gsp }} L$ when the current density of the electron beam is small. The profile becomes higher and narrower when the stimulated emission increases.

$D_{\text {gsp }}$ ranges from 0 to 1 . However, the maximum value of $D_{\text {st }}$ becomes very small when the electron spreading length $\ell$ is short.

Numerical examples of the optical output profile $|F(L)|^{2}$ in Eq. (16) are shown in Fig. 5. The parameters in this figure were chosen to fit the experimental values given in Sec. IV. When the current density $J$ of the electron beam is small, the profiles are given with the term $D_{\mathrm{gsp}} L$ as the guided spontaneous emission because the value of $g-\alpha_{\text {loss }}$ is not sufficiently large. When the current density increases, the output profile becomes higher and narrower due to the stimulated emission represented by the gain coefficient $g$.

\section{EXPERIMENTAL OBSERVATION}

\section{A. Design of the waveguide}

The optical waveguide should be made of materials with high refractive indices that are transparent to optical light. We used a silicon-on-insulator (SOI) substrate as the waveguide, which is used for integrated circuit technology and consists of $\mathrm{Si}-\mathrm{SiO}_{2}-\mathrm{Si}$ layers. Our target wavelength for optical emission is $\lambda \approx 1.5 \mu \mathrm{m}$. The core layer of the waveguide is the top Si layer, whose thickness is $d=0.32 \mu \mathrm{m}$ and refractive index is $n_{1}=3.485$. The second layer is $\mathrm{SiO}_{2}$, whose thickness is $1.0 \mu \mathrm{m}$ and refractive index is $n_{2}$ $=1.444$ and which is used as the cladding layer. The third layer is a Si bulk substrate, whose thickness is more than $300 \mu \mathrm{m}$ and which holds the waveguide together. The second $\mathrm{SiO}_{2}$ layer is sufficiently thick to confine the optical field to around the top Si layer, preventing leakage in the direction of the third Si bulk substrate. Another cladding layer is of course the vacuum space, whose refractive index is $n_{0}=1.0$.

We did not carry out any fabrication along the width $(x)$ direction in this experiment. The spatial width of the guided optical light is determined by the width of the electron beam.

Although the field distribution $\boldsymbol{T}(x, y)$ of the electricfield components and the propagation constant $\beta$ are mainly

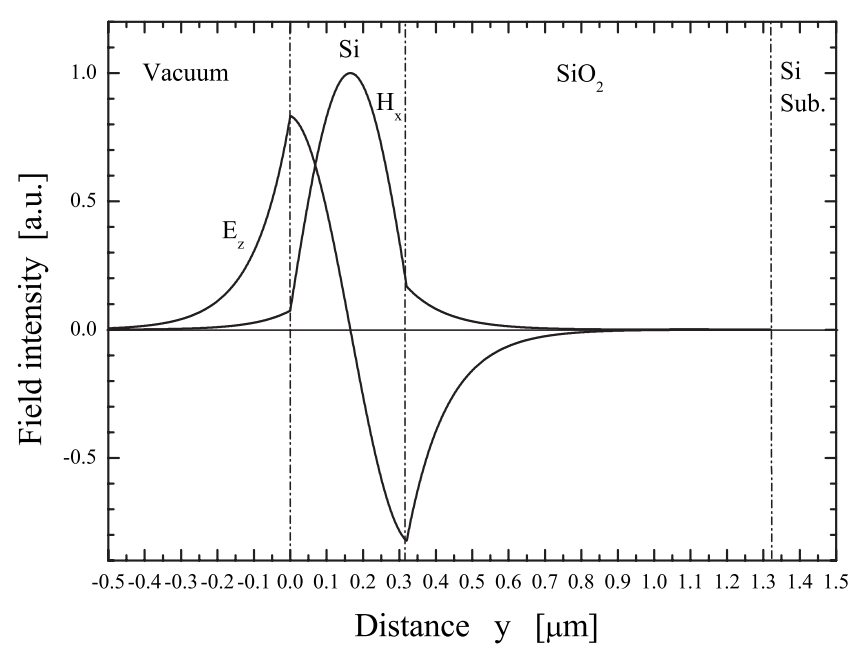

FIG. 6. Example of the transverse-field distribution for a fundamental TM mode in the slab waveguide. The optical emission is generated by the electric component $E_{z}$ in the vacuum region.

analyzed using Eq. (7), it is more convenient to analyze $H_{x}$ by using the following equation for the TM modes:

$$
\left(\frac{\partial^{2}}{\partial y^{2}}-\beta^{2}+\mu_{0} \varepsilon_{i} \omega^{2}\right) H_{x}=0,
$$

where the integer $i$ indicates the layer number. The remaining electric-field components are given by

$$
\begin{aligned}
& E_{y}=\frac{1}{j \omega \varepsilon_{i}} \frac{\partial H_{x}}{\partial z}=-\frac{\beta}{\omega \varepsilon_{i}} H_{x}, \\
& E_{z}=-\frac{1}{j \omega \varepsilon_{i}} \frac{\partial H_{x}}{\partial y} .
\end{aligned}
$$

An example of the transverse-field distribution for a fundamental TM mode is shown in Fig. 6. The optical emission is generated by the electric component $E_{z}$ in the vacuum region.

Since the transverse-field distribution varies with the wavelength $\lambda$, the effective refractive index $n_{\text {eff }}$ also varies with $\lambda$. Thus, the condition $v_{e}=v_{\text {opt }}$ depends on the wavelength as well as the acceleration voltage $V$. Calculated examples of the relations between the wavelength $\lambda$ and the acceleration voltage $V$ satisfying the condition $v_{e}=v_{\mathrm{opt}}$ are shown in Fig. 7. The solid circles in this figure are the experimentally observed wavelengths of the optical emission, as will be explained in Sec. IV B.

\section{B. Experiment}

The experimental setup is illustrated in Fig. 8. An electron gun and a waveguide were installed in a vacuum chamber. The electron accelerating voltage was varied in the range of $30-50 \mathrm{kV}$ and the emission current flow into the cathode was approximately $50 \mu \mathrm{A}$.

The optical waveguide was set on a mechanical manipulator in the chamber to control the position and angle with respect to the electron beam. Optical output was observed through a view port and was detected by an InGaAs detector 


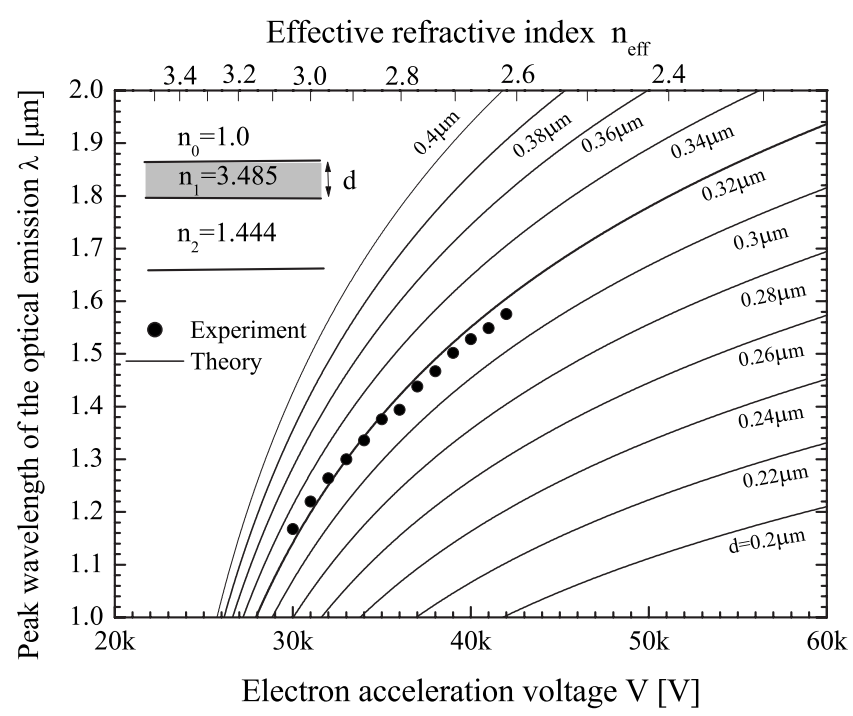

FIG. 7. Relation between the wavelength $\lambda$ of the optical emission and the electron acceleration voltage $V$ required to achieve the condition $v_{e}=v_{\text {opt }}$. Lines show the theoretically calculated data, while solid circles show the experimentally obtained data.

after passing through a polarizer and a monochromator. The TM and TE modes were selected by adjusting the direction of the polarizer to $E_{y}$ and $E_{x}$, respectively.

The width of the electron beam was 200-300 $\mu \mathrm{m}$, which is much larger than the spreading width of the guided light into the vacuum region. The spreading width was expected to be in the range from 0.1 to $0.5 \mu \mathrm{m}$ as shown in Fig. 6. Thus, the optical emission must be obtained under the condition that the electron beam touches the optical waveguide.

The waveguide was made of a SOI substrate whose main body was made of Si crystal. We connected the back surface of the SOI substrate to the earth to remove charged electrons.

The position of the electron beam was monitored by two television cameras through view ports because the waveguide emitted an almost blue light when the electron beam touched the waveguide. This blue-light emission appeared to originate from the $\mathrm{SiO}_{2}$ layer and was clearly generated by a phenomenon different from that of our proposed emission at $\lambda \approx 1.5 \mu \mathrm{m}$.

The electron beam could propagate almost $10 \mathrm{~mm}$ along the surface of the waveguide and was absorbed in the SOI substrate. We thus estimate the length of the device to be $L$ $=10 \mathrm{~mm}$.

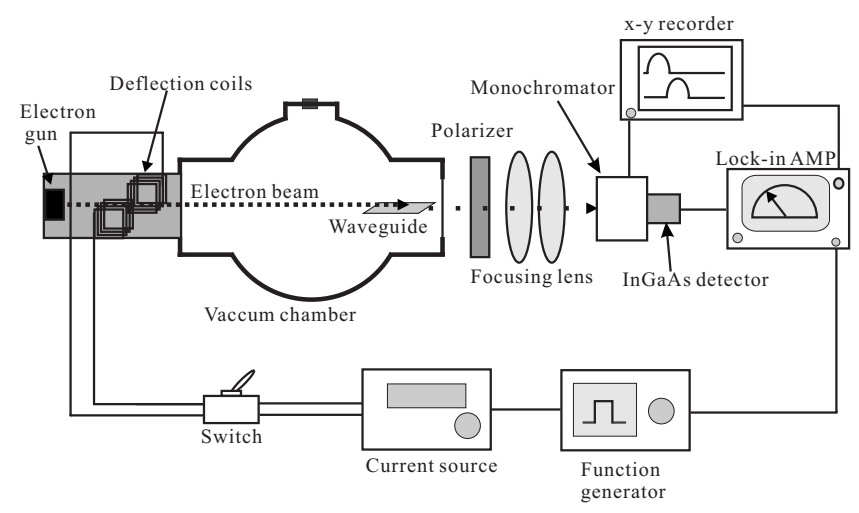

FIG. 8. Configuration of the experimental setup for optical emission.

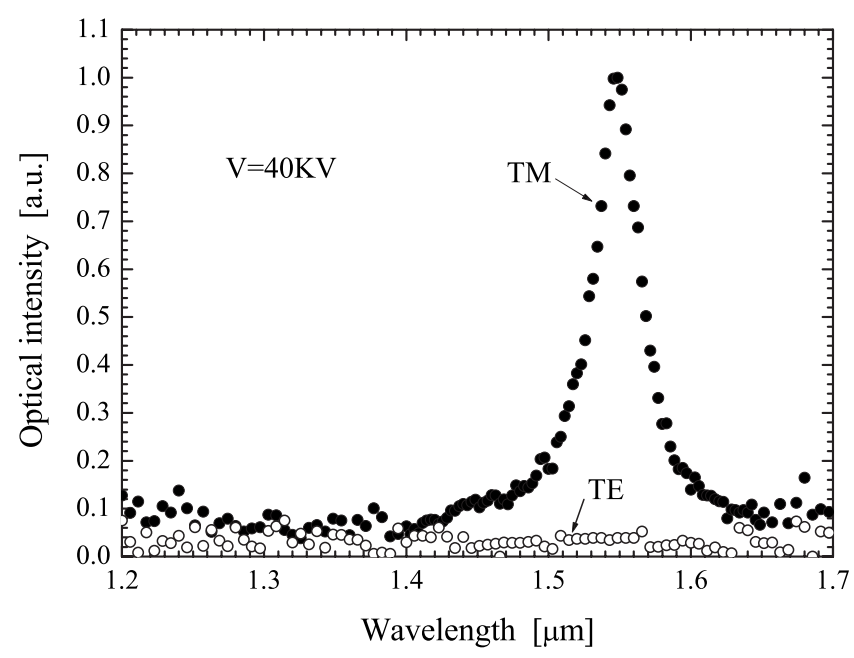

FIG. 9. Modal dependence of the optical emission. The optical emission was observed in the TM mode but not in the TE mode.

We used the lock-in technique to detect the optical emission. The position of the electron beam was changed by applying a rectangular current to the deflection coils in the electron gun. The repetition ratio of the rectangular current was $40 \mathrm{~Hz}$. The electron beam touched the waveguide during half-period intervals of $12.5 \mathrm{~ms}$, and was removed from the waveguide in the other half periods of $12.5 \mathrm{~ms}$. Then the optical emission was detected by reducing unnecessary disturbances using a lock-in amplifier.

Examples of the measured spectrum are shown in Fig. 9 for an electron acceleration voltage of $40 \mathrm{kV}$. The solid circles in this figure represent data obtained in the TM mode and the open circles represent data obtained in the TE mode. It is clear that the optical emission was observed in the TM mode but not in the TE mode. The peak wavelength was $1.55 \mu \mathrm{m}$. Thus, the optical light must be transparent to the Si crystal.

Optical spectra for different acceleration voltages $V$ are shown in Fig. 10. The emitting wavelength was shifted to a longer wavelength with increasing acceleration voltage. The variation in the peak wavelength of the optical emission with

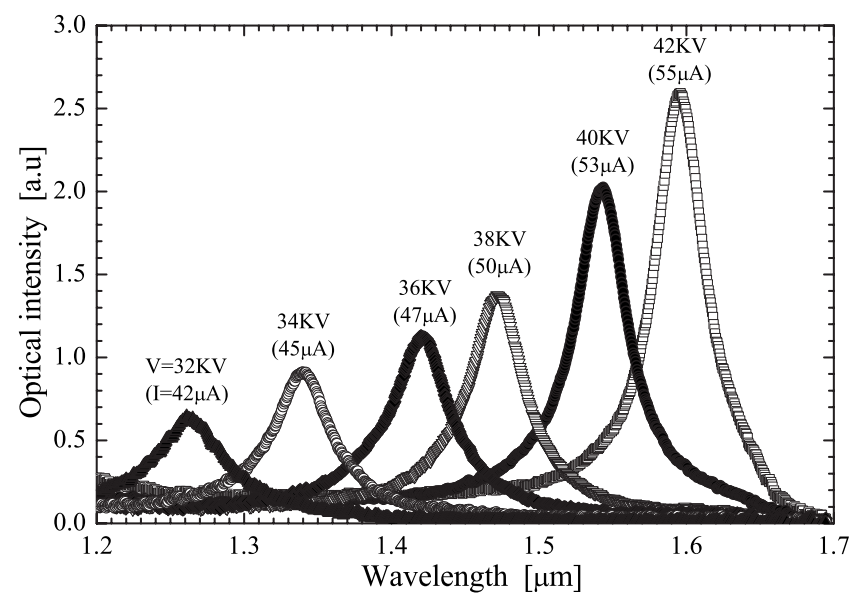

FIG. 10. Optical spectrum for different acceleration voltages $V$. The emission current is indicated in the parentheses. The optical spectrum shifted to a longer wavelength with increasing acceleration voltage. 


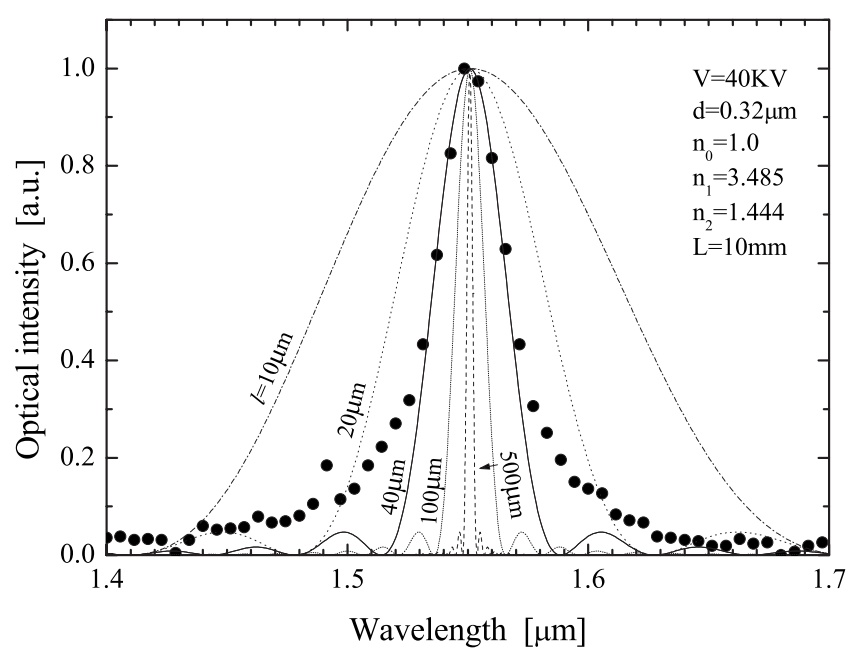

FIG. 11. Optical emission spectrum including comparison of the experimental data with theoretical calculations. The spreading length of an electron is estimated to be $\ell \approx 40 \mu \mathrm{m}$.

the acceleration voltage has already been shown in Fig. 7 with solid circles. The experimentally obtained results coincide well with the theoretically calculated data.

We did not measure the current of the electron beam directly but monitored the emission current $I$ that flowed into the cathode of the electron gun. The maximum allowable emission current was different for each acceleration voltage. The spectra in Fig. 10 were obtained, with the maximum emission current $I$ shown in parentheses.

We also examined the variation in the optical spectrum with the emission current at a fixed acceleration voltage. The peak intensity of the optical emission increased with increasing emission current, but the width of the spectrum did not changed markedly within the range of our experiment.

\section{COMPARISON BETWEEN EXPERIMENT AND THEORY}

The features of the experimentally obtained data coincided well with our theoretical predictions. Examples of this are the limitation of the polarization to the TM mode and the variation in the peak wavelength of the optical emission with the acceleration voltage $V$. These results support the validity of the present scheme for optical emission.

However, another aim for us is to determine the parameters characterizing the emission mechanism. When the optical gain $g$ is not sufficiently large, the property of the optical emission is mostly determined by the term of the guided spontaneous emission $C_{\mathrm{gsp}}$. The calculated values of $C_{\mathrm{gsp}}$ in Eq. (30) and $D_{\text {gsp }}$ in Eq. (31) are shown in Fig. 11 with the spreading length or the coherent length $\ell$ of an electron as a parameter. The experimentally recorded emission spectrum is plotted with solid circles.

This comparison indicates that the spreading length of an electron is $\ell \approx 40 \mu \mathrm{m}$. This value almost coincides with the electron spacing given as $1 / N^{1 / 3}$ for the electron density of $N \approx 1.5 \times 10^{13} \mathrm{~m}^{-3}$, which corresponds to the current density $J \approx 280 \mathrm{~A} / \mathrm{m}^{2}$ with electron velocity $v_{e} \approx c / n_{\mathrm{eff}} \approx 1$ $\times 10^{8} \mathrm{~m} / \mathrm{s}$, which gives an electron-beam current of 8.9-20 $\mu \mathrm{A}$ for a beam width of 200-300 $\mu \mathrm{m}$. Here, the value of the beam width was obtained from the specifications of the electron gun. Since the electron-beam current is lower than the emission current $I$ in the cathode, such a numerical evaluation is acceptable. Clearly, the spreading length of $\ell$ $\approx 40 \mu \mathrm{m}$ can be regarded as the coherent length of the electron wave, which is determined by the static Coulomb forces among electrons.

The relaxation time $\tau$ must be determined by the dynamics of the electrons, such as electron-to-electron collisions caused by the differences in velocity of the electrons. The distribution of the electron velocity is estimated to be $\Delta v_{e}$ $\approx 860 \mathrm{~m} / \mathrm{s}$ for the cathode temperature $T \approx 2000 \mathrm{~K}$ in the electron gun. Thus, the relaxation time is estimated to be $\tau$ $\approx 10^{-7} \mathrm{~s}$. The mean free path between two consecutive collisions is $v_{e} \tau \approx 10 \mathrm{~m}$. This value is much larger than the spreading length $\ell$ of an electron.

The above-mentioned situation is very different from the case of a semiconductor crystal, in which an electron can spread over a wide range beyond the size determined by $1 / N^{1 / 3}$ and the spatial superposition of electron waves is possible because the neutral electric charge condition exists due to the presence of positive charges in the crystal. Furthermore, the coherent length $\ell$ in a semiconductor crystal is mostly determined by the electron collisions. ${ }^{26}$

We could not confirm the effect of the stimulated emission in this experiment. The reason seems to be the low electron current density. The stimulated emission at $100 \mathrm{GHz}$ has already been reported for a similar configuration to that used by us, ${ }^{19,20}$ but the electron density in that experiment was almost 100 times larger than that in our experiment. We expect that the effect of the stimulated emission can be clarified by increasing the electron-beam current density by more than two orders of magnitude.

\section{COMPARISON WITH CLASSICAL THEORY}

There are many classical analyses in the literature on the interaction between a traveling electron and an electromagnetic wave including those based on the Cherenkov laser. ${ }^{2,11-18,27-31}$ The term "classical" in this section means that the electron is regarded as a spatially localized point particle. On the other hand, "quantum mechanical treatment" means to regard the electron as a wave spreading in space, whose spreading range is the size of an electron as well as the coherent length of an electron wave.

In typical classical theories on Cherenkov radiation, the emission of an electromagnetic wave in a uniformly expanding space is analyzed. On the other hand, our model in this paper is applied to a waveguide structure. Thus, a direct comparison between our analysis and the classical analysis seems to be difficult but we will attempt to make a comparison as follows.

We suppose here that an electron moves with constant velocity $v_{e}$ along the $z$-axis in a uniformly expanding material with refractive index $n_{1}$. The energy of the radiated electromagnetic wave is given by Eq. (3.8.31) of Ref. 17 as 


$$
\begin{aligned}
\frac{d^{2} E(\omega, \theta)}{d \omega d \Omega}= & \frac{\mu_{0} e^{2} v_{e}^{2} n_{1}}{4 \pi c} \sin ^{2} \theta \\
& \times\left|\frac{\omega}{2 \pi} \int_{-T}^{T} e^{-j \omega t\left\{1-v_{e}\left(n_{1} / c\right) \cos \theta\right\}} d t\right|^{2} \\
= & \frac{\mu_{0} e^{2} v_{e}^{2} n_{1}}{4 \pi c} \sin ^{2} \theta\left(\frac{\omega T}{\pi}\right)^{2} \\
& \times \operatorname{Sinc}^{2}\left[T \omega\left(1-\frac{v_{e} n_{1} \cos \theta}{c}\right)\right],
\end{aligned}
$$

where $\theta$ is the angle between the directions of the radiated electromagnetic wave and the electron motion, $\Omega$ is the solid angle around the radiated direction, $\omega$ is the angular frequency of the electromagnetic wave, and $2 T$ is the time interval in which the electron radiates the electromagnetic wave. The term $\left(n_{1} \omega / c\right) \cos \theta$ is the $z$-component of the wavevector of the radiated electromagnetic wave.

In the case of the optical waveguide in this paper, the spatial angles $\theta$ and $\Omega$ should be fixed in restricted values. Then, the effective refractive index $n_{\text {eff }}$ is introduced as

$$
\frac{n_{1}}{c} \cos \theta=\frac{n_{\mathrm{eff}}}{c} .
$$

The profile of the spectrum of the radiated electromagnetic wave is given by the function $\operatorname{Sinc}^{2}[x]$ in Eq. (38) and is rewritten as

$$
D_{\text {class }}=\operatorname{Sinc}^{2}\left[T \omega\left(1-\frac{n_{\mathrm{eff}} v_{e}}{c}\right)\right] .
$$

On the other hand, the spectrum profile in our treatment was given in Eq. (31). By supposing the energy conservation rule between the electron motion and the electromagnetic wave under the nonrelativistic condition, we can rewrite the profile in terms of the velocity by using the relation of

$$
\begin{aligned}
E_{b}-E_{a} & =\frac{\hbar^{2}\left(k_{b}^{2}-k_{a}^{2}\right)}{2 m_{0}} \approx \frac{\hbar^{2} k_{b}\left(k_{b}-k_{a}\right)}{m_{0}}=v_{e} \hbar\left(k_{b}-k_{a}\right) \\
& \equiv \hbar \omega
\end{aligned}
$$

to

$$
D_{\mathrm{gsp}}=\operatorname{Sinc}^{2}\left[\frac{\ell \omega}{2 v_{e}}\left(1-\frac{n_{\mathrm{eff}} v_{e}}{c}\right)\right] .
$$

We found here that the interaction time interval $2 T$ in the classical treatment is related to the time required for the electron particle to move the length of the electron wave $\ell$ by $2 T=\ell / v_{e}$. Since, by comparison between the experimental data and our theoretical analysis, we estimated that $\ell$ $\approx 40 \mu \mathrm{m}$, the interaction time is $2 T \approx 4.0 \times 10^{-13} \mathrm{~s}$ $=0.4$ ps when $v_{e} \approx 1 \times 10^{8} \mathrm{~m} / \mathrm{s}$.

\section{CONCLUSION}

A scheme proposed for optical emission was theoretically and experimentally confirmed. The emission is generated by a traveling electron beam and a high-refractive-index optical waveguide in a vacuum environment. The condition for obtaining optical emission and amplification was that the velocity of the electron coincided with the phase velocity of the optical field. An optical field with an electric component along the direction of the traveling electron can be generated.

The generating mechanism of the optical field is called the guided spontaneous emission, while the amplification is generated by the stimulated emission. We realized this emission at a wavelength of approximately $1.5 \mu \mathrm{m}$ using a $\mathrm{Si}$ thin film and an electron gun with an acceleration voltage between 30 and $50 \mathrm{kV}$.

The value of the spreading length or the coherent length of an electron wave is an important parameter in evaluating the optical emission and amplification. The spreading length of the electron wave was found to coincide with the electron spacing in the vacuum environment.

We could not confirm the effect of the stimulated emission in the current experiment. Confirming the effect of stimulated emission by increasing the current density of the electron beam might be the focus of a future study.

\section{ACKNOWLEDGMENTS}

The authors thank Professor M. Asada of Tokyo Institute of Technology for detailed discussions on the proposed scheme. They also thank Mr. Y. Kakimoto, a valued staff member at Kanazawa University, for constructing the experimental setup. This research was supported by the Ministry of Education, Culture, Sports, Science and Technology of Japan through a scientific grant-in-aid.

${ }^{1}$ J. R. Pierce and L. M. Field, Proc. IRE 35, 108 (1947).

${ }^{2}$ J. R. Pierce, Proc. IRE 35, 111 (1947).

${ }^{3}$ H. Motz, J. Appl. Phys. 22, 527 (1951).

${ }^{4}$ D. A. G. Deacon, Phys. Rev. Lett. 38, 892 (1977).

${ }^{5}$ J. E. Walsh, T. C. Marshall, and S. P. Schlesinger, Phys. Fluids 20, 709 (1977).

${ }^{6}$ J. Soln, J. Appl. Phys. 54, 2036 (1983).

${ }^{7}$ E. Garate, R. Cook, P. Heim, R. Layman, and J. Walsh, J. Appl. Phys. 58, 627 (1985).

${ }^{8}$ J. Walsh, in High Power Microwave Sources, edited by V. L. Granastein and I. Aexeff (Artech, Boston, MA, 1987), p. 421.

${ }^{9}$ W. Main and R. Cherry, Appl. Phys. Lett. 55, 1498 (1989).

${ }^{10}$ F. Ciocci, A. Doria, G. P. Gallerano, I. Giabbai, M. F. Kimmitt, G. Messina, and A. Renieri, Phys. Rev. Lett. 66, 699 (1991).

${ }^{11}$ W. Case, in High Power Microwave Sources, edited by V. L. Granastein and I. Aexeff (Artech, Boston, MA, 1987), p. 412.

${ }^{12}$ J. E. Walsh and J. B. Murphy, IEEE J. Quantum Electron. 18, 1259 (1982).

${ }^{13}$ T. Shiozawa and H. Kondo, IEEE J. Quantum Electron. 23, 1633 (1987).

${ }^{14}$ E. Fisch, A. K. Henning, and J. Walsh, IEEE J. Quantum Electron. 27, 753 (1991).

${ }^{15}$ T. Shiozawa and H. Kamata, IEEE J. Quantum Electron. 33, 1687 (1997).

${ }^{16}$ I. J. Owens and J. H. Brownell, Phys. Rev. E 67, 036611 (2003).

${ }^{17}$ T. Shiozawa, Classical Relativistic Electrodynamics (Springer-Verlag, Berlin, 2004).

${ }^{18}$ H. L. Andrews and C. A. Brau, J. Appl. Phys. 101, 104904 (2007).

${ }^{19}$ E. E. Fisch and J. E. Walsh, Appl. Phys. Lett. 60, 1298 (1992).

${ }^{20}$ I. J. Owens and J. H. Brownell, J. Appl. Phys. 97, 104915 (2005).

${ }^{21}$ N. Miyabe, A. Ikeda, M. Asakawa, M. Kusaba, and Y. Tsunawaki, Proceedings of FEL 2007, Novosibirsk, Russia, p. 406, 2007.

${ }^{22}$ M. Yamada, IEEE J. Quantum Electron. 35, 147 (1999).

${ }^{23}$ M. Asada and M. Yamada, J. Appl. Phys. 95, 5123 (2004).

${ }^{24}$ Y. Kuwamura, M. Yamada, Y. Ichino, I. Fukamura, and T. Sekikawa, Jpn. J. Appl. Phys. 43, 7109 (2004).

${ }^{25}$ D. Marcuse, Principles of Quantum Electronics (Academic, New Jersey, 1980).

${ }^{26}$ M. Yamada, H. Ishiguro, and H. Nagato, Jpn. J. Appl. Phys. 19, 135 (1980).

${ }^{27}$ I. F. Frank and I. E. Tamm, C. R. Acad. Sci. URSS 14, 109 (1937). 
${ }^{28}$ J. D. Jackson, Classical Electrodynamics (Wiley, New York, 1962).

${ }^{29}$ W. K. H. Panofsky and M. Phillips, Classical Electricity and Magnetism, 2nd ed. (Addison-Wesley, Massachusetts, 1962).
${ }^{30}$ L. D. Landau and E. M. Lifshiz, Electrodynamics of Continuous Media (Pergamon, Oxford, 1960).

${ }^{31}$ A. Yariv and C. C. Shih, Opt. Commun. 24, 233 (1978). 